\title{
ANÁLISE DO GRAU DE RESTRITIVIDADE DE EXIGÊNCIAS TÉCNICAS ÀS EXPORTAÇÕES BRASILEIRAS
}

\author{
Rosane Nunes de Farial \\ Heloisa Lee Burnquist ${ }^{2}$
}

\begin{abstract}
Resumo - O objetivo deste trabalho foi avaliar o grau de restritividade das exigências técnicas às exportações brasileiras, a partir de resultados de uma pesquisa conduzida com 77 empresas exportadoras. As variáveis detalhadas no trabalho representam seis tipos de medidas técnicas usualmente requeridas para produtos exportados. Com a finalidade de reduzir o número de variáveis a serem analisadas e facilitar a interpretação, utilizou-se a técnica de análise fatorial, que permitiu agregar as variáveis e construir dois indicadores: o Indicador de Especificação Técnica (IET) e o Indicador de Informação e Rotulagem (IIR). As estatísticas descritivas e o teste t emparelhado mostraram que as empresas entrevistadas consideram o IET mais restritivo às suas exportações, ou seja, elas têm sofrido maiores restrições por aspectos na confecção do produto, abrangendo sua qualidade, os padrões no processo de produção e testes de avaliação de conformidade.
\end{abstract}

Palavras-chave: exigências técnicas, survey, exportações brasileiras, indicadores de restritividade.

\section{Introdução}

As barreiras técnicas vêm tomando formas diversas e apresentam-se como um desafio cada vez maior para o crescimento do comércio internacional. Segundo Josling et al. (2002), é consenso que o fluxo de comércio sofre impactos da imposição de regulamentos técnicos, e o número crescente de disputas no âmbito da Organização Mundial do Comércio (OMC) sinaliza que esse tipo de medida regulatória vem

Doutoranda em Economia Aplicada pela ESALQ/USP. E-mail: rnfaria@esalq.usp.br

Professora do Departamento de Economia, Administração e Sociologia da Escola Superior de Agricultura "Luiz de Queiroz", Universidade de São Paulo. E-mail: hlburnqu@esalq.usp.br 
assumindo importância crescente no delineamento dos fluxos comerciais. Henson e Loader (2001) avaliam que as medidas técnicas podem reduzir os fluxos de comércio, tanto pelo aumento dos custos de produção e de marketing para a adequação às exigências quanto pela dificuldade de as empresas entrarem e permanecerem em novos mercados. Em algumas instâncias, os custos podem ser indiretos, decorrentes da necessidade de redirecionar o produto a outros mercados menos dinâmicos.

A despeito da percepção crescente e da existência de um Acordo sobre Barreiras Técnicas às Exportações, no âmbito da Organização Mundial do Comércio, desde 1995, a mensuração dos efeitos de medidas técnicas sobre o comércio internacional ainda se constitui em um desafio para economistas e formuladores de política econômica.

Embora já se identifique a percepção das empresas quanto à importância dos impactos das exigências técnicas ao comércio internacional, as informações necessárias para a condução de trabalhos empíricos encontram-se relativamente dispersas e pouco organizadas. Claramente, torna-se necessário implantar um sistema de levantamento das informações, para que estas possam ser trabalhadas e adequadas à análise econômica. Essas informações podem auxiliar o setor produtivo e o setor público a se guiarem com maior clareza para a implementação de políticas que visem reduzir os impactos negativos das medidas técnicas sobre o comércio internacional.

O presente trabalho apresenta resultados de uma iniciativa nesse sentido, conduzida em empresas exportadoras brasileiras. Os dados levantados foram organizados e submetidos a análises estatísticas, buscando mensurar o grau de restritividade das exigências técnicas às exportações brasileiras, por meio de indicadores. $\mathrm{O}$ estudo foi desenvolvido para mostrar a viabilidade da construção desses indicadores e fundamentar a proposição de que a sua atualização ao longo do tempo proporcionaria uma referência importante para formuladores de políticas. Os indicadores obtidos são úteis para discernir onde devem ser concentrados esforços para amenizar os efeitos das barreiras técnicas ao comércio. 
O trabalho está organizado da seguinte forma: uma breve discussão sobre as medidas técnicas e as pesquisas relacionadas é apresentada nos itens 2 e 3. Em seguida são apresentados os métodos estatísticos e o questionário. No item 5 são apresentados os resultados, e as conclusões são discutidas no item 6 .

\section{Barreiras técnicas ao comércio}

Para Roberts e DeKremer (1997), as barreiras técnicas podem ser definidas como:

Standards governing the sale of products in national markets which have as their prima facie objective the correction of market inefficiencies stemming from externalities associated with the production, distribution and consumption of these products.

Em outras palavras, barreiras técnicas ao comércio podem ser caracterizadas em uma situação em que um produtor/exportador precisa alterar seu produto para cumprir as exigências feitas pelos parceiros comerciais, a fim de assegurar aspectos relacionados à saúde, segurança, ambiente, bem como aspectos de proteção ao consumidor. Esses requisitos podem ser impostos por instituições públicas, no caso das regulamentações técnicas, e por organizações não-governamentais, em se tratando de barreiras não-regulatórias e normas. $\mathrm{O}$ caráter legal da regulamentação a distingue das normas; as últimas são voluntárias e comumente - embora não seja uma regra - são desenvolvidas pela iniciativa privada, sendo de interesse de produtores e consumidores envolvidos na produção e consumo do bem. Podem ser úteis para facilitar o fluxo de informação nas transações comerciais, bem como para assegurar a compatibilidade entre produtos. A regulamentação geralmente relaciona-se a especificações técnicas ou testes e exigência de certificação, que asseguram que o produto atende às particularidades que a ele são impostas (avaliação de conformidade). 
As barreiras técnicas têm-se mostrado importantes para as operações de negócios envolvendo pré-produção, produção, vendas e política de marketing, pois podem afetar o comércio de maneira mais restritiva do que a proibição parcial de importações através de instrumentos tradicionais de protecionismo comercial, como as tarifas ou quotas (Josling et al., 2002). Exigências técnicas que induzem à necessidade de adequar o design de um produto, reorganizar o sistema de produção e realizar testes múltiplos e certificações podem onerar a exportação a ponto de torná-la proibitiva. Nesse caso, a exigência torna-se uma barreira técnica ao comércio.

Esses mesmos autores destacam que as exigências técnicas podem ser impostas tanto para produtos quanto para processos de produção. As exigências a produtos se referem principalmente às suas características, como tamanho, peso, composição química, aspectos da embalagem e outros atributos. Quando exigências dessa natureza são impostas a um determinado bem, as firmas podem manter inalterado o processo de produção do bem, visando minimizar seus custos e comprovar, por meio de testes, que seu produto está de acordo com as exigências.

As exigências nos processos, no entanto, estão relacionadas diretamente à tecnologia de produção das firmas. Como exemplo, pode-se destacar a adoção dos sistemas Hazard Analysis and Critical Control Point (HACCP) e System Approach. Nesse caso, as firmas arcam com os custos de adequação dos processos, mas podem ser poupadas dos testes nos carregamentos.

Antle (1996), citada por Josling et al. (2002), argumenta que as exigências para produtos são mais eficientes como ferramentas regulatórias, pois permitem às firmas escolherem a tecnologia de produção que minimiza seus custos e, concomitantemente, atende aos objetivos dos regulamentos.

Josling et al. (2002) argumentam que as exigências nos processos de produção impõem um problema fundamental para a política comercial, uma vez que o monitoramento e a verificação do cumprimento envolvem sistemas administrativos dos dois países em questão. Também alertam 
que a crescente importância atribuída pelos consumidores à questão da qualidade do produto tem feito com que aumente o monitoramento desses atributos por meio de exigências no processo.

\section{Pesquisas relacionadas}

Beghin e Bureau (2001) apresentaram vários métodos que podem ser empregados para avaliar o impacto de medidas técnicas ao comércio. Dentre eles, destaca-se o Survey. Os autores enfatizam que os questionários aplicados diretamente às empresas tornam possível conhecer as medidas mais restritivas e focar nas questões mais relevantes. Os questionários podem ser aplicados para prover informações como, por exemplo, o ranking de importância medido em uma escala. As informações coletadas podem ser utilizadas a posteriori em análises econométricas ou análises multivariadas.

$\mathrm{Na}$ literatura internacional encontram-se vários trabalhos nessa linha. OECD (2000) conduziu um survey com 55 firmas em três setores nos Estados Unidos, Japão, Reino Unido e Alemanha, buscando identificar as medidas mais restritivas em cada um deles. Os resultados encontrados indicaram que, enquanto o setor de leite e derivados julga os requerimentos dos clientes como medidas técnicas mais restritivas, o setor de equipamentos de telecomunicação considera os requerimentos legais e o processo de avaliação de conformidade os fatores mais restritivos.

Henson et al. (2000) realizaram um survey juntamente com entrevistas estruturadas em países em desenvolvimento para investigar as dificuldades em cumprir as normas e regulamentos técnicos dos países desenvolvidos.

Wilson e Otsuki (2004) desenvolveram um survey para investigar globalmente o impacto de requerimentos técnicos em países em desenvolvimento. O objetivo foi levantar informações, em nível de firmas, sobre a intensidade com que as medidas técnicas interferem na habilidade 
de exportar os produtos. As informações coletadas em 689 firmas, em 17 países em desenvolvimento, constituíram um banco de dados, do Banco Mundial, sobre barreiras técnicas.

No Brasil esse tipo de pesquisa ainda é escasso, e nessa linha destacamse os trabalhos de Ferraz Filho (1997), que procurou, por meio de questionário, investigar a incidência de barreiras não-tarifárias às exportações brasileiras, e Kume et al. (2001), que, mediante a aplicação de questionários, identificaram e avaliaram as principais barreiras nãotarifárias do Mercosul.

Como o Brasil não participou da pesquisa do Banco Mundial, este trabalho visou adicionar e atualizar informações sobre exigências técnicas em nível de firmas.

\section{Metodologia}

\subsection{O questionário}

O questionário foi formulado a partir dos trabalhos conduzidos por Wilson e Otsuki (2004) e OCDE (2000). Para avaliar o grau de restritividade das exigências técnicas, as empresas classificaram seis tipos de exigências segundo o grau de interferência em suas exportações, utilizando para isso uma escala de Likert, que varia de 1 a 5 . Nessa escala, considerase que 1 indica nenhuma interferência e 5 indica alta interferência das exigências nas exportações.

Os seis tipos de exigências técnicas avaliados pelas empresas e utilizados como variáveis nas análises são:

$X_{1}=$ exigência no processo de produção;

$\mathrm{X}_{2}=$ exigência quanto à embalagem do produto;

$\mathrm{X}_{3}=$ exigência quanto à rotulagem do produto; 
$\mathrm{X}_{4}=$ exigência de testes de avaliação de conformidade;

$\mathrm{X}_{5}=$ exigência de especificações de qualidade; e

$\mathrm{X}_{6}=$ exigências ambientais.

\subsection{A amostra}

Para seleção da amostra utilizou-se o catálogo de exportadores brasileiros disponível no site da Confederação Nacional das Indústrias - CNI. Foram selecionadas empresas de todos os Estados brasileiros que exportaram acima de US\$1 milhão de reais no biênio 2004/05. Foram contatadas, aleatoriamente, por telefone 246 empresas, das quais 77 retornaram o questionário.

\subsection{Método estatístico}

\subsubsection{Análise Fatorial}

A análise fatorial de dados permite reduzir o número original de variáveis, de tal forma que os fatores extraídos possam explicar as variáveis originais. Esse método teve início com os trabalhos de Karl Pearson em 1901 e Charles Spearman em 1904, durante um estudo para a criação de um índice de inteligência. Em 1947, Thurstone retomou a idéia dos precursores e desenvolveu a análise fatorial. Desde então, ela tem sido amplamente empregada em diferentes áreas da ciência (Reis, 1997). 
Segundo Johnson e Wichern (2002), o modelo fatorial para a i-ésima variável pode ser expresso algebricamente da seguinte forma: ${ }^{3}$

$$
Y_{i}-\mu_{i}=l_{i 1} F_{1}+l_{i 2} F_{2}+\ldots+l_{i m} F_{i m}+\varepsilon_{i}
$$

em que $\mu_{i}$ é a média da variável i; $F_{j}$ representa o j-ésimo fator comum não-correlacionado, como média zero e variância unitária; $l_{i j}$ é o fator de carga da i-ésima variável no j-ésimo fator; e ${ }^{\varepsilon_{i}}$ é o termo de erro, com média zero, que capta a variação específica em $Y_{i}$ não explicada pelos fatores comuns.

O modelo fatorial implica a seguinte estrutura de covariâncias para a iésima variável:

$$
\begin{aligned}
& \operatorname{Var}\left(Y_{i}\right)=l_{i 1}^{2}+l_{i 2}^{2}+\ldots+l_{i m}^{2}+\Psi_{i} \\
& \operatorname{Cov}\left(Y_{i}, Y_{k}\right)=l_{i 1} l_{k 1}+\ldots+l_{i m} l_{k m} \\
& \operatorname{Cov}\left(Y_{i}, F_{j}\right)=l_{i j} \quad i=1,2, \ldots, p \quad \text { e } \quad j=1,2, \ldots, m
\end{aligned}
$$

em que a porção $\left(l_{i 1}^{2}+l_{i 2}^{2}+\ldots+l_{i m}^{2}\right)$ explicada pelos $m$ fatores comuns é chamada de i-ésima comunalidade (indica a proporção da variância total da variável que é explicada pelo conjunto de fatores comuns) e a porção $\Psi_{i}$ é chamada de variância específica. 
As estimativas das cargas fatoriais podem ser obtidas por vários métodos, porém neste trabalho empregou-se o método mais comum, que são os componentes principais, e utilizou-se a rotação ortogonal Varimax.

Para testar a adequação do modelo de análise fatorial, geralmente utilizase a estatística de Kaiser-Meyer-Olkin (KMO) e o teste de esfericidade de Bartlett. O KMO é um indicador que compara a magnitude dos coeficientes de correlação observados com as magnitudes dos coeficientes de correlação parcial e varia entre 0 e 1 . Pequenos valores de KMO (abaixo de 0,5) indicam que o uso da análise fatorial não é adequado. Por sua vez, o teste de esfericidade de Bartlett serve para testar a hipótese nula de que a matriz de correlação é uma matriz identidade. Se essa hipótese não for rejeitada, o uso do modelo de análise fatorial deve ser reavaliado.

Na determinação do número de fatores necessários para representar o conjunto de dados, considera-se a contribuição individual e adicional de cada fator para a variância explicada do conjunto de dados. Usualmente, são considerados apenas os fatores cuja raiz característica é maior que a unidade, isto é, aqueles que correspondem a uma proporção da variância superior àquela atribuída a uma variável isolada.

Após a extração dos fatores, é possível criar indicadores que sumarizam a informação dada pelos itens que os integram (Pestana e Gageiro, 2000). Esses indicadores são resultantes de médias ponderadas pelas cargas fatoriais da matriz de componentes rotacionados. Considerando o j-ésimo fator, o indicador é calculado de acordo com a equação a seguir. 


$$
I_{j}=\frac{\sum_{i=1}^{p} l_{i j} Y_{i}}{\sum_{i=1}^{p} l_{i j}} \quad i=1,2, \ldots, p \quad \text { e } p \text { número de variáveis }
$$

Neste trabalho, os indicadores são usados para mensurar o grau de restritividade das exigências técnicas às exportações brasileiras.

\section{Análise dos resultados}

\subsection{Caracterização das empresas participantes da pesquisa}

Das empresas colaboradoras, $50 \%$ são do setor de agronegócio, o que parece compatível com o perfil exportador do País. O conjunto de empresas dos setores de química, mineração, metalurgia e siderurgia representou o segundo maior percentual, compondo $28 \%$ do total. Já os outros setores específicos, que são o automotivo e o de máquinas e equipamentos, tiveram participação similar: $9 \%$ cada um.

Verificou-se que 55\% das empresas participantes eram de capital nacional, sendo as restantes de capital multinacional. Quanto aos países de destino dos principais produtos exportados pelas empresas, verificou-se que os países em desenvolvimento apresentaram ligeira predominância entre as empresas colaboradoras $(31,9 \%)$, quando comparados aos mercados de economia desenvolvida $(27,7 \%)$. O maior percentual $(40,4 \%)$ direcionava suas exportações para os dois destinos (países desenvolvidos e em desenvolvimento). 


\subsection{Resultados da análise fatorial}

Primeiramente, foram avaliadas as notas atribuídas pelas empresas ao grau de interferência das exigências técnicas em suas exportações. A Tabela 1 apresenta a média e o desvio-padrão do grau de interferência para cada exigência

Tabela 1 - Média do grau de interferência das exigências às exportações atribuídas por 77 empresas pesquisadas - junho, julho e agosto de 2006

\begin{tabular}{lcc}
\hline \multicolumn{1}{c}{ Tipo de Exigências } & Média & Desvio-padrão \\
\hline $\mathrm{X}_{1}$ - exigência no processo de produção & 3,21 & 1,268 \\
$\mathrm{X}_{2}$ - exigência quanto à embalagem do produto & 2,71 & 1,081 \\
$\mathrm{X}_{3}$ - exigência quanto à rotulagem do produto & 2,09 & 1,048 \\
$\mathrm{X}_{4}$ - exigência de testes de avaliação de conformidade & 3,28 & 1,207 \\
X $_{5}$ - exigência de especificações de qualidade & 3,62 & 1,113 \\
X $_{6}$ - exigência ambiental & 2,97 & 1,177 \\
\hline
\end{tabular}

Fonte: Resultado da pesquisa.

As exigências referentes à qualidade aparecem com maior média de interferência nas exportações. Os fatores seguintes, por ordem de importância, são as exigências de testes de conformidade e exigências no processo, respectivamente. Às exigências de rotulagem, por sua vez, foram atribuídos os menores graus de interferência, quando comparados aos graus dos demais tipos de exigências técnicas. Wilson e Otsuki (2004) encontraram resultados semelhantes em pesquisa realizada para o Banco Mundial envolvendo países em desenvolvimento. Aparentemente, medidas consideradas mais restritivas às exportações por empresas em países como Argentina, Nigéria, Índia, entre outros, também são as mais restritivas às exportações brasileiras.

A técnica de análise fatorial foi empregada com o objetivo de reduzir o número de variáveis analisadas e, assim, facilitar a compreensão do fenômeno, tendo em vista que a realização das análises subseqüentes torna-se mais fácil quando se trabalha com poucos fatores. 
Para análise de adequação dos dados à técnica, fez-se o teste de esfericidade de Bartlett, que foi significativo a 1\%, rejeitando a hipótese nula de que a matriz de correlação é uma matriz identidade. $O$ teste de KMO apresentou valor de 0,805. Segundo Pestana e Gageiro (2000), valores entre 0,7 e 0,8 permitem classificar a adequação como mediana. Assim, ambos os testes realizados permitiram concluir que a amostra de dados é passível de ser analisada pela técnica de análise fatorial.

A Tabela 2 mostra que a análise pelos componentes principais identificou uma raiz característica com valor superior a 1 .

Tabela 2 - Raízes características da matriz de correlação, proporção e proporção acumulada da variância explicada pelos fatores

\begin{tabular}{lccc}
\hline Fatores & $\begin{array}{c}\text { Raiz } \\
\text { característica }\end{array}$ & \% da variância & \% acumulada \\
\hline 1 & 3,442 & 57,36 & 57,36 \\
2 & 0,913 & 15,21 & 72,58 \\
3 & 0,642 & 10,69 & 83,28 \\
4 & 0,447 & 7,45 & 90,73 \\
5 & 0,343 & 5,71 & 96,44 \\
6 & 0,213 & 3,55 & 100,00 \\
\hline
\end{tabular}

Fonte: Resultados da pesquisa.

Para a interpretação, optou-se por utilizar dois fatores, tendo em vista que o segundo fator possui raiz próxima de 1 e contribui com $15 \%$ da variância total. Os dois captam uma proporção significativa da variância total das variáveis originais: $72,58 \%$.

Com o objetivo de melhorar a leitura e interpretação dos dois fatores, foi feita uma rotação pelo método Varimax, mantendo a ortogonalidade entre eles. Na Tabela 3 são apresentadas as cargas fatoriais, que são os coeficientes de correlação entre cada fator e cada uma das seis variáveis, após a rotação. Na última coluna desta tabela encontra-se o valor da comunalidade, que é a proporção da variância da variável que é explicada pelos dois fatores identificados. Na última linha da mesma tabela encontra- 
se a proporção da variância total explicada por cada fator. A análise das cargas fatoriais forneceu uma interpretação ou um significado aos dois fatores (Tabela 3).

Tabela 3 - Cargas fatoriais, comunalidades e percentagem da variância total correspondente a cada fator, após a rotação

\begin{tabular}{lcrc}
\hline \multirow{2}{*}{ Variáveis } & \multicolumn{2}{c}{ Carga Fatorial } & \multirow{2}{*}{ Comunalidade } \\
\cline { 2 - 3 } & $\mathbf{F}_{\mathbf{1}}$ & $\mathbf{F}_{\mathbf{2}}$ & \\
\hline $\mathrm{X}_{1}$ - exigência no processo de produção & $\mathbf{0 , 8 3 6}$ & 0,262 & 0,767 \\
X $_{2}$ - exigência quanto à embalagem do produto & 0,219 & $\mathbf{0 , 8 1 2}$ & 0,708 \\
X $_{3}$ - exigência quanto à rotulagem do produto & 0,200 & $\mathbf{0 , 8 5 6}$ & 0,773 \\
X $_{4}$ - exigência de testes de conformidade & $\mathbf{0 , 8 4 9}$ & 0,205 & 0,764 \\
X $_{5}$ - exigência de especificações de qualidade & $\mathbf{0 , 8 7 9}$ & 0,255 & 0,838 \\
X $_{6}$ - exigência ambiental & 0,483 & $\mathbf{0 , 5 2 2}$ & 0,506 \\
Percentagem da variância & 41,907 & 30,683 & \\
\hline
\end{tabular}

Fonte: Resultados da pesquisa.

O primeiro fator $\left(\mathrm{F}_{1}\right)$ explica $41,90 \%$ da variabilidade total e encontra-se forte e positivamente correlacionado com: exigência no processo de produção $\left(X_{1}\right)$, exigência de testes de conformidade $\left(X_{4}\right)$ e exigência de especificações de qualidade do produto $\left(\mathrm{X}_{5}\right)$. Em resumo, o $\mathrm{F}_{1}$ pode ser interpretado como uma medida do grau de interferência das exigências técnicas relacionadas diretamente às especificações técnicas.

$\mathrm{O}$ segundo fator $\left(\mathrm{F}_{2}\right)$ explica $30,68 \%$ da variabilidade total e associa-se forte e positivamente com: exigência quanto à embalagem do produto $\left(\mathrm{X}_{2}\right)$, exigência quanto à rotulagem do produto $\left(\mathrm{X}_{3}\right)$ e exigência ambiental $\left(\mathrm{X}_{6}\right)$. Ou seja, esse fator pode ser interpretado como uma medida do grau de interferência das exigências técnicas relacionadas diretamente à informação e rotulagem.

Como as variáveis usadas no modelo fatorial foram medidas por meio de uma escala de Likert, utilizou-se o Alpha de Cronbach para verificar a consistência interna dos fatores. $\mathrm{O}$ resultado para $\mathrm{F}_{1}$ foi de 0,873 , o que 
indica boa consistência interna deste fator; já para $\mathrm{F}_{2} \mathrm{o}$ valor do Alpha de 0,712 indica média consistência interna.

Após obtenção dos fatores e das cargas fatoriais, foram calculados dois indicadores, interpretados segundo a sua composição. Conforme apresentado na Equação (5), o primeiro indicador foi calculado a partir dos valores das cargas fatoriais de $\mathrm{F}_{1}$. Portanto, é composto essencialmente por medidas relacionadas à qualidade, exigências no processo e testes de avaliação de conformidade (variáveis que têm maior peso na composição do indicador). Dessa forma, foi denominado Indicador de Especificação Técnica - IET, pois está diretamente relacionado às especificações técnicas, incluindo normas e regulamentos de produtos e de processos, como, por exemplo, normas no método de produção, especificação de ingredientes e restrição de aditivos. De forma análoga, realizou-se o cálculo do segundo derivado de $\mathrm{F}_{2}$, composto principalmente pelas exigências de embalagem e de rotulagem, juntamente com exigências ambientais, o qual foi denominado Indicador de Informação e Rotulagem - IIR, pois está mais relacionado à provisão de informações aos consumidores, visando reduzir o risco associado ao produto e aumentar a eficiência na sua utilização, uma vez que assegura que o consumidor sabe o que o produto contém e se foi desenvolvido atendendo a requisitos de não-agressão ao ambiente.

Os indicadores foram utilizados em outras análises estatísticas, a fim de verificar o grau de restritividade das exigências técnicas nas exportações brasileiras. 


\subsubsection{Análises estatísticas dos indicadores}

A Tabela 4 apresenta as estatísticas descritivas dos dois indicadores calculados a partir dos fatores extraídos.

Tabela 4 - Estatísticas descritivas dos indicadores

\begin{tabular}{|c|c|c|}
\hline Variáveis & Média & Desvio-padrão \\
\hline IET & 3,20 & 0,953 \\
\hline IIR & 2,74 & 0,843 \\
\hline
\end{tabular}

Fonte: Resultados da pesquisa.

Observa-se que em média o IET é superior ao IIR, o que permite inferir que as exportações das empresas entrevistadas têm sido mais impactadas por aspectos de confecção do produto do que por aspectos de informação e embalagem. A fim de verificar se as médias são estatisticamente diferentes, utilizou-se o teste $\mathrm{t}$ emparelhado. O teste de KolmogorovSminorv para normalidade mostrou que os indicadores são normais a $1 \%$ de significância estatística, o que viabiliza a utilização do teste $t$ emparelhado. Neste teste, a hipótese nula é de que a média da diferença emparelhada (sendo o par igual a IET - IIR) é zero e a hipótese alternativa é que a média da diferença é diferente de zero.

O teste foi significativo a $1 \%$, podendo-se concluir que a média da diferença $(0,462)$ é estatisticamente diferente de zero, indicando que o Indicador de Especificações Técnicas (IET) é mais restritivo às exportações das empresas brasileiras do que o Indicador de Informação e Rotulagem (IIR).

O fato de o IET, que engloba exigência nos processos e testes de avaliação de conformidade (que também podem estar relacionados aos processos), apresentar-se mais restritivo que o IIR corrobora, em parte, os argumentos de Josling et al. (2002), de que as exigências relacionadas aos processos são mais restritivas que aquelas relacionadas aos produtos. 
O IIR, que engloba as medidas relacionadas à rotulagem e embalagem ambientais, apresentou grau de interferência menor nas exportações. As questões relacionadas à rotulagem são as que menos interferem nas exportações das empresas.

Em geral, os resultados indicaram uma direção para a implementação de políticas públicas e pesquisas no sentido de auxiliar as empresas no cumprimento das exigências. Isso sugere que questões de qualidade e exigências nos processos merecem atenção especial no planejamento da exportação. Investimentos nessa área são essenciais, em face da tendência de valorização dos atributos de qualidade pelos consumidores. Conseqüentemente, a imposição de exigências nos processos também pode ser uma prática cada vez mais aplicada pelos países importadores.

\section{Conclusão}

A pesquisa aplicada mostrou ser possível identificar, com significância estatística, a percepção das empresas exportadoras brasileiras pesquisadas quanto à importância dos obstáculos técnicos ao comércio internacional, o que se considera relevante para fins de planejamento de ações voltadas às exportações, seja pelo setor privado ou pelo setor público. Isso é importante, pois ressalta que, embora as ações de políticas comerciais ainda não caracterizem os obstáculos técnicos às exportações do País como prioridade, o comércio vem sendo afetado de forma significativa por tais medidas. De fato, a importância das restrições de natureza técnica ao comércio internacional pôde ser expressa por meio de dois indicadores que mensuram o grau de restritividade das exigências técnicas às exportações brasileiras: o Indicador de Especificações Técnicas (IET) e o Indicador de Informação e Rotulagem (IIR).

Um segundo aspecto relevante a ser observado é que, para as 77 empresas envolvidas na pesquisa, o IET apresenta-se mais elevado. Ou seja, aparentemente, ações tomadas no sentido de mitigar restrições técnicas às exportações brasileiras terão impacto positivo mais expressivo 
se voltadas para aspectos da produção. Fatores referentes à comercialização e distribuição do produto são também importantes, mas podem apresentar menor impacto, se as exigências de estágios anteriores concentrados na produção não forem priorizadas.

Pode-se inferir, portanto, que a adequação às restrições técnicas que aparentemente vêm prejudicando de forma significativa as exportações do País, de forma mais próxima à produção que à comercialização e distribuição dos produtos, deve estimular a competitividade, promovendo a facilitação das trocas com outros países. É interessante, portanto, que as empresas sejam estimuladas a prover informações e a interagir com representantes legais e,ou, instituições públicas, para avaliar as vantagens e desvantagens de adequações e capacitações promovidas pelas exigências técnicas apresentadas pelos países importadores. Uma participação mais efetiva das autoridades governamentais no estabelecimento de Acordos de Reconhecimento Mútuo, que facilita a equivalência das medidas adotadas, bem como a maior cooperação técnica entre os países, também pode ser um fator positivo.

Considera-se ainda que a avaliação por segmentos específicos de exportação pode vir a se constituir em um avanço importante para a identificação de maneiras para minimizar os impactos negativos dessas medidas para os fluxos de comércio. Para isso, a obtenção e organização de informações em bancos de dados torna-se um requisito essencial. 


\section{Referências}

ANDERSON, T. W. An introduction to multivariate statistical methods. 2. ed. New York: John Wiley, 1984.

BEGHIN, J. C.; BUREAU, J. C. Quantitative policy analysis of sanitary, phytosanitary and technical barriers to trade. Économie Internationale, v. 87, p. 107-130, 2001.

FERRAZ FILHO, G. Barreiras técnicas ao comércio internacional: a experiência das exportações brasileiras. Revista Brasileira de Comércio Exterior, n. 52, p. 47-56, 1997.

HENSON, S.; LOADER, R. Barriers to agricultural exports from developing countries: the role of sanitary and phytosanitary requirements. World Development, v. 29, n. 1, p. 85 - 102, 2001.

HENSON, S.; LOADER, R.; SWINBANK, A.; BREDAHL, M.; LUX, $\mathrm{N}$. Impact of sanitary and phytosanitary measures on developing countries. The University of Reading, Department of Agricultural and Food Economics, 2000, 106 p.

KUME, H.; ANDERSON, P.; OLIVEIRA JUNIOR, M. Identificação das barreiras ao comércio no mercosul: a percepção das empresas exportadoras brasileiras. Planejamento e Políticas Públicas, n. 23, 2001, 40p.

JOHNSON R. A.; WICHERN D. W. Applied multivariate statistical analysis, 5. ed. New Jersey: Prentice Hall, 2002. 767 p.

JOSLING, T.; ROBERTS, D.; ORDEN, D. Food regulations and trade: toward a safe and open global system (Draft Form). Washington, USA: Institute for International Economics, 2002. 
MORRISON, D. F. Multivariate statistical methods. 2. ed. New York: McGraw-Hill, 1976.

OECD. An assessment of the costs for international trade in meeting regulatory requirements. Working Party of the Trade Committee, 2000, 102p.

PESTANA, M. H.; GAGEIRO, J. N. Análise de dados para ciências sociais - a complementaridade do SPSS. 2 ed. Lisboa: Edições Sílabo, 2000.569 p.

REIS, E. Estatística multivariada aplicada. Lisboa: Edições Silabo, 1997.

ROBERTS, D.; DeKREMER, K. Technical barriers to US agricultural exports. Washington, DC: Economic Research Service, USDA, 1997.

WEYERBROCK, S.; XIA, T. Technical trade barriers in US/Europe agricultural trade. Agribusiness, v.16, n.2, p. 235-251, 2000.

WILSON, J. S.; OTSUKI, T. Standards and technical regulations and firms in developing countries: new evidence from a world bank technical barriers to trade survey (Preliminary Draft). Washington, USA: The World Bank, 2004. 55p. 


\begin{abstract}
The objective of this investigation was to evaluate to what degree technical requirements restrict Brazilian exports, based on a survey applied to 77 firms. The variables represented six types of technical measures that are usually imposed upon exported products. To facilitate the interpretation of the results, factorial analysis was used to reduce the number of variables involved. This allowed the construction of two indicators of the degree of restrictiveness of the technical barriers focused by the study. These were an indicator of requirements of technical specification (ITS) and an indicator for information and label requirements (IIL). Descriptive statistics and paired $t$ test showed that the firms surveyed considered the ITS more restrictive to their exports, that is, these have been subject to greater restrictions affecting the production process, including quality, the production process pattern and conformity assessment.
\end{abstract}

Key-words: technical requirements, survey, Brazilian exports, restrictiveness indicators. 